\title{
Analysis of IECC $(2003,2006$, 2009) and ASHRAE 90.1-2007 Commercial Energy Code Requirements for Mesa, AZ
}

Y Huang

K Gowri

February 2011
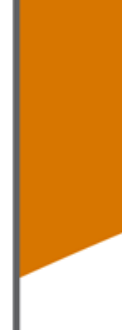

\section{Pacific Northwest}

NATIONAL LABORATORY

Proudly Operated by Battelle Since 1965 


\title{
DISCLAIMER
}

This report was prepared as an account of work sponsored by an agency of the United States Government. Neither the United States Government nor any agency thereof, nor Battelle Memorial Institute, nor any of their employees, makes any warranty, express or implied, or assumes any legal liability or responsibility for the accuracy, completeness, or usefulness of any information, apparatus, product, or process disclosed, or represents that its use would not infringe privately owned rights. Reference herein to any specific commercial product, process, or service by trade name, trademark, manufacturer, or otherwise does not necessarily constitute or imply its endorsement, recommendation, or favoring by the United States Government or any agency thereof, or Battelle Memorial Institute. The views and opinions of authors expressed herein do not necessarily state or reflect those of the United States Government or any agency thereof.

\author{
PACIFIC NORTHWEST NATIONAL LABORATORY \\ operated by \\ BATTELLE \\ for the \\ UNITED STATES DEPARTMENT OF ENERGY \\ under Contract DE-AC05-76RL01830
}

Printed in the United States of America
Available to DOE and DOE contractors from the Office of Scientific and Technical Information,
P.O. Box 62, Oak Ridge, TN 37831-0062; ph: (865) 576-8401
fax: (865) 576-5728
email: reports@adonis.osti.gov

\begin{abstract}
Available to the public from the National Technical Information Service, U.S. Department of Commerce, 5285 Port Royal Rd., Springfield, VA 22161 ph: (800) 553-6847 fax: (703) 605-6900

email: orders@ntis.fedworld.gov

online ordering: http://www.ntis.gov/ordering.htm
\end{abstract}

This document was printed on recycled paper. 
Analysis of IECC (2003, 2006, 2009) and ASHRAE 90.1-2007

Commercial Energy Code Requirements for Mesa, AZ

Y Huang

K Gowri

February 2011

Prepared for

U.S. Department of Energy

under Contract DE-AC05-76RL01830

Pacific Northwest National Laboratory

Richland, Washington 99352 


\section{Executive Summary}

This report summarizes code requirements and energy savings for commercial buildings in climate zone 2B built to the 2009 IECC and ASHRAE Standard 90.1-2007 when compared to the 2003 IECC and the 2006 IECC.

In general, the 2009 IECC and ASHRAE Standard 90.1-2007 have higher insulation requirements for exterior walls, roof, and windows and have higher efficiency requirements for heating, ventilating and air-conditioning (HVAC) equipment. HVAC equipment efficiency requirements are governed by the National Appliance Energy Conservation Act of 1987 (NAECA), and are applicable regardless of the IECC version adopted.

The energy analysis results show that commercial buildings meeting the 2009 IECC requirements save between $4.4 \%$ and $9.5 \%$ site energy and between $4.1 \%$ and $9.9 \%$ energy cost when compared to the 2006 IECC; and save between 10.6\% and 29.4\% site energy and between $10.3 \%$ and $29.3 \%$ energy cost when compared to the 2003 IECC. Similar analysis comparing ASHRAE Standard 90.1-2007 requirements to the 2006 IECC shows that the energy savings are between $4.0 \%$ and $10.7 \%$ for multifamily and retail buildings, but less than $2 \%$ for office buildings. Further comparison of ASHRAE Standard 90.1-2007 requirements to the 2003 IECC shows site energy savings in the range of $7.7 \%$ to $30.6 \%$ and an energy cost savings ranging from 7.9\% to 30.3\%. Both the 2009 IECC and ASHRAE Standard 90.1-2007 have the potential to save energy by comparable levels for most building types. 


\section{Table of Contents}

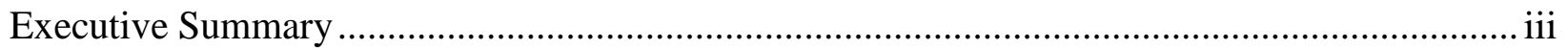

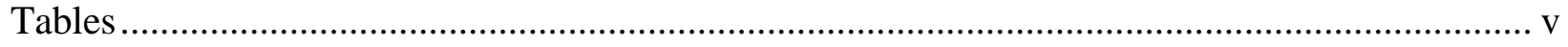

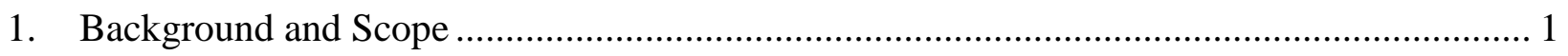

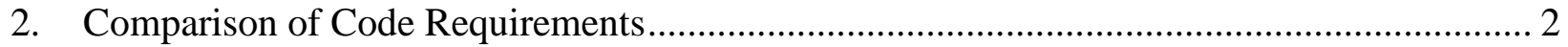

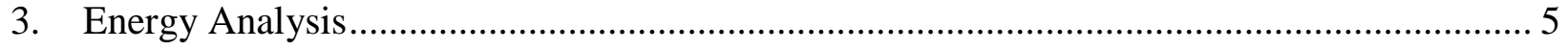

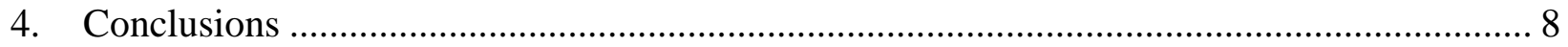

Appendix A - Prototype Building Descriptions ............................................................ 9 


\section{Tables}

Table 1: Comparison of Envelope Requirements ............................................................... 3

Table 2: Comparison of Mechanical and Lighting Requirements ............................................. 4

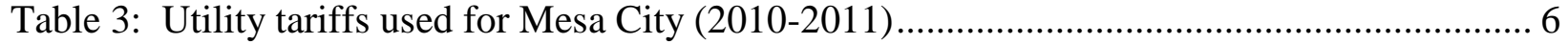

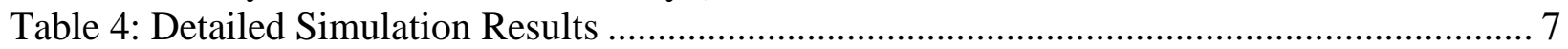

Table 5: Energy Cost Summary (\$/sf/year) ........................................................................... 7

Table 6: Energy Use and Cost Savings Comparison (percentage) ............................................. 8

Table 7: $\mathrm{CO}_{2}$ Emission Reduction Comparison (percentage) ................................................... 8 


\section{Background and Scope}

The city of Mesa, AZ is considering adoption of 2009 IECC/ASHRAE Standard 90.1-2007 and requested the assistance of the Department of Energy's (DOE's) Building Energy Codes Program to determine energy savings and environmental impact of adopting the 2009 IECC/ASHRAE Standard 90.1-2007 compared to the 2003 and 2006 IECC. Because Mesa currently has not adopted any energy code and most new construction is based on the 2006 IECC, both 2003 IECC and 2006 IECC are used as baselines to compare the impact of 2009 IECC and ASHRAE 90.1-2007. The 2003 IECC baseline is included in this study to represent buildings that are less efficient and do not meet the 2006 IECC.

The following four building types have been chosen for energy simulation and analysis:

1. Multifamily residential: Mid-rise apartment building

2. Nonresidential: Small three-story office building

3. Nonresidential: Small retail building

4. Semiheated: Industrial office/warehouse building

DOE Commercial Reference Building Models ${ }^{\mathrm{a}}$ for the above building types were adapted for the analysis. Four sets of energy simulation using EnergyPlus were carried out to estimate energy consumption for each building model meeting the envelope, lighting and mechanical requirements of the 2003 IECC, the 2006 IECC, the 2009 IECC and ASHRAE Standard 90.1-2007.

This report focuses on the comparison of code requirements and energy analysis results for the above building types. In addition to energy savings estimates, the analysis also includes annual energy cost and $\mathrm{CO}_{2}$ emissions estimates. This study does not address cost effectiveness analysis or payback period for the additional cost of complying with the newer codes.

\footnotetext{
${ }^{a}$ http://www1.eere.energy.gov/buildings/commercial_initiative/reference_buildings.html
} 


\section{Comparison of Code Requirements}

The commercial code requirements in the 2003 IECC, the 2006 IECC, the 2009 IECC and ASHRAE Standard 90.1-2007 for envelope, mechanical and lighting systems are listed in Tables 1 and 2. All code requirements for climate zone 2B from the 2006 IECC, 2009 IECC and ASHRAE Standard 90.1-2007, and climate zone 3C from the 2003 IECC are used in this comparison. The scope of comparison is limited to building characteristics that can be modeled for detailed energy simulation and analysis. Major differences are summarized below:

- Insulation requirements for roofs and above-grade walls in the 2009 IECC are significantly more stringent for all construction types when compared to the 2003/2006 IECC.

- Both the 2009 IECC and ASHRAE Standard 90.1-2007 have separate sets of requirements for residential (Group "R") and non-residential buildings. However, the insulation requirements for residential and non-residential construction in the 2009 IECC are the same except for mass walls.

- Below-grade wall insulation and slab insulation requirements remain the same in all codes except for the 2009 IECC, which requires a more stringent F-factor (F-0.54) for a residential unheated slab.

- The 2003 IECC has no requirements for window U-factors, and solar heat gain coefficient (SHGC) requirements were less stringent compared to the 2006 IECC, the 2009 IECC and ASHRAE Standard 90.1-2007.

- Metal building insulation requirements in the 2009 IECC are more stringent, and mandatory continuous insulated sheathing on metal building walls is required.

- Plastic skylight requirements are removed in the 2009 IECC, and all skylights are required to meet more stringent U-factor and SHGC requirements.

- HVAC equipment efficiency requirements for unitary air conditioners, chilled water systems and boilers are more stringent in the 2009 IECC and ASHRAE Standard 90.1-2007 (these are based on National Appliance Energy Conservation Act (NAECA) requirements and are applicable regardless of the version of IECC adopted).

- Economizer requirements in the 2009 IECC require economizers when cooling capacity exceeds 54 $\mathrm{kBtu} / \mathrm{h}$.

- Interior lighting power allowances remain the same in all codes for the area categories of buildings analyzed in this study, although there are variations for some space types.

- Lighting zone based power allowance requirements are specified for exterior lighting in the 2009 IECC and ASHRAE Standard 90.1-2007.

A detailed comparison of code requirements in the 2009 IECC and ASHRAE Standard 90.1-2007 can be found at: http://www.energycodes.gov/publications/research/documents/codes/90-1_iecc_comparison_final_12-162009.pdf 
Table 1: Comparison of Envelope Requirements

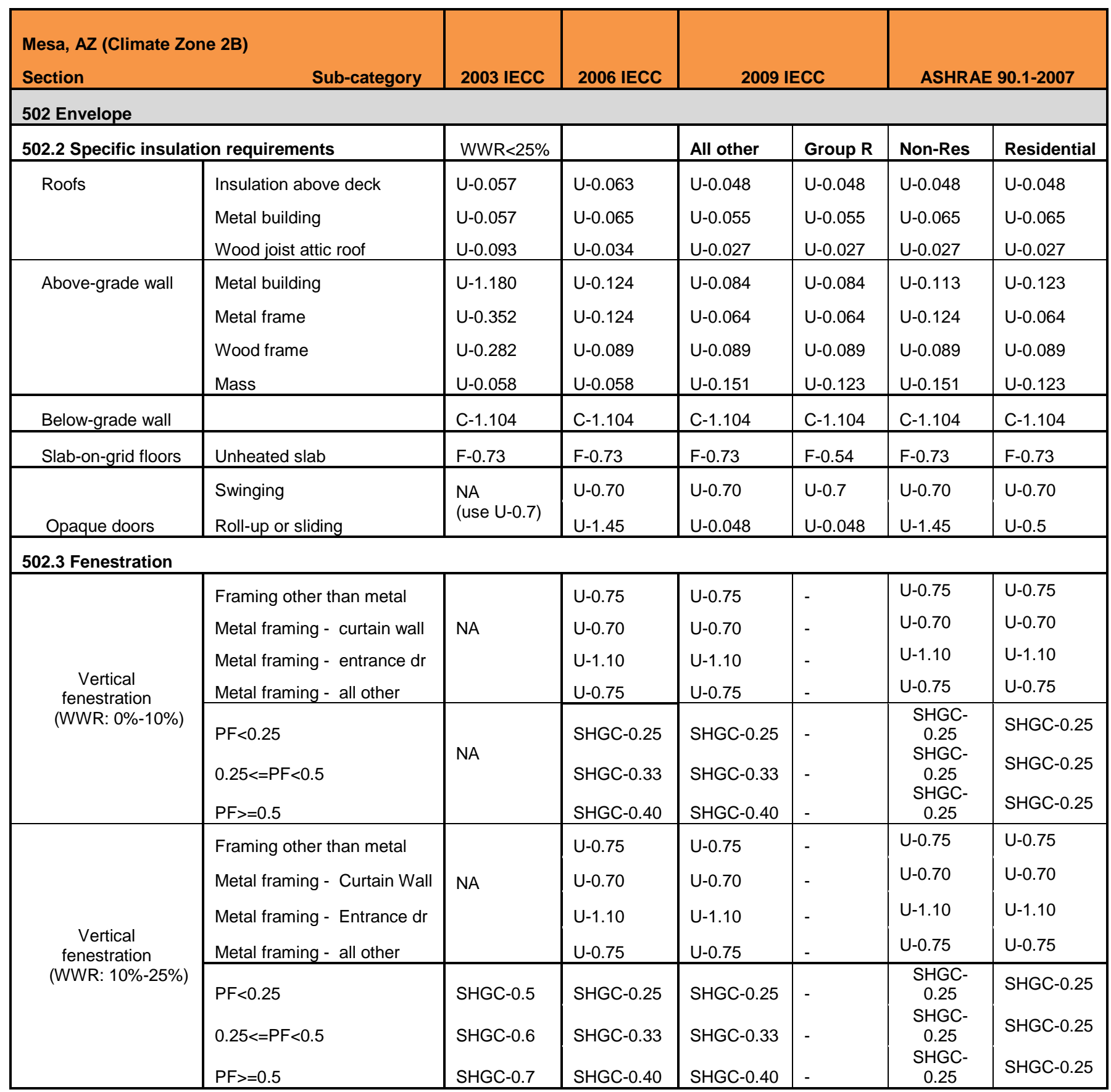


Table 2: Comparison of Mechanical and Lighting Requirements

\begin{tabular}{|c|c|c|c|c|c|}
\hline \multicolumn{2}{|c|}{ Mesa, AZ (Climate Zone 2B) } & \multirow[b]{2}{*}{2003 IECC } & \multirow[b]{2}{*}{2006 IECC } & \multirow[b]{2}{*}{2009 IECC } & \multirow[b]{2}{*}{ ASHRAE 90.1-2007 } \\
\hline Section & Sub-category & & & & \\
\hline \multicolumn{6}{|c|}{503 Mechanical } \\
\hline \multicolumn{6}{|c|}{ 503.2.3 HVAC equipment performance requirements } \\
\hline \multirow{6}{*}{$\begin{array}{l}\text { Unitary AC, } \\
\text { Electrical }\end{array}$} & $<65 \mathrm{kBtu} / \mathrm{h}$ Single package & 10.0 SEER & 10.0 SEER & 13.0 SEER & 13.0 SEER \\
\hline & $<65$ kBtu/h Split & 9.7 SEER & 9.7 SEER & 13.0 SEER & 13.0 SEER \\
\hline & $>=65 \mathrm{kBtu} / \mathrm{h} \&<135 \mathrm{kBtu} / \mathrm{h}$ & N.A & 10.3 EER & 11.2 EER & 11.2 EER \\
\hline & $>=135 \mathrm{kBtu} / \mathrm{h} \&<240 \mathrm{kBtu} / \mathrm{h}$ & & 9.7 EER & 11.0 EER & 11.0 EER \\
\hline & $>=240 \mathrm{kBtu} / \mathrm{h} \&<760 \mathrm{kBtu} / \mathrm{h}$ & & 9.5 EER & 10.0 EER & 10.0 EER \\
\hline & $>760 \mathrm{kBtu} / \mathrm{h}$ & & 9.2 EER & 9.7 EER & 9.7 EER \\
\hline $\begin{array}{l}\text { Warm air } \\
\text { furnaces }\end{array}$ & Gas fired & $80 \% \mathrm{Et}$ & $80 \% \mathrm{Et}$ & $80 \% \mathrm{Et}$ & $80 \% \mathrm{Et}$ \\
\hline DCV & $\begin{array}{l}\text { space }>500 \text { sqft }+40 \\
\text { person/1000sqft }\end{array}$ & NA & NA & Required & Required \\
\hline ERV & $\begin{array}{l}\text { Fan }>=5000 \mathrm{cfm}+70 \% \mathrm{~min} \\
\text { fraction }\end{array}$ & NA & ERV+exceptions & ERV+exceptions & ERV+exceptions \\
\hline \multicolumn{6}{|c|}{ 503.2.10 Air System design and control } \\
\hline & fan motor $>5 \mathrm{hp}$ & NA & NA & $\begin{array}{l}\text { CAV: } h p<= \\
\text { CFM*0.0011 } \\
\text { VAV: } h p<= \\
\text { CFM*0.0015 }\end{array}$ & $\begin{array}{l}\text { CAV: } \mathrm{hp<=} \\
\text { CFM*0.0011 } \\
\text { VAV: } \mathrm{hp<=} \\
\text { CFM }^{*} 0.0015\end{array}$ \\
\hline \multicolumn{6}{|l|}{505 Lighting } \\
\hline \multicolumn{6}{|c|}{505.5 Interior lighting power requirements (use building type overall LPD) } \\
\hline & Multifamily building & $0.7 \mathrm{w} / \mathrm{ft}^{2}$ & $0.7 \mathrm{w} / \mathrm{ft}^{2}$ & $0.7 \mathrm{w} / \mathrm{ft}^{2}$ & $0.7 \mathrm{w} / \mathrm{ft}^{2}$ \\
\hline & Retail & $1.5 \mathrm{w} / \mathrm{ft}^{2}$ & $1.5 \mathrm{w} / \mathrm{ft}^{2}$ & $1.5 \mathrm{w} / \mathrm{ft}^{2}$ & $1.5 \mathrm{w} / \mathrm{ft}^{2}$ \\
\hline & $\begin{array}{l}\text { Warehouse } \\
\text { Office }\end{array}$ & $\begin{array}{l}0.8 \mathrm{w} / \mathrm{ft}^{2} \\
1.0 \mathrm{w} / \mathrm{ft}^{2}\end{array}$ & $\begin{array}{l}0.8 \mathrm{w} / \mathrm{ft}^{2} \\
1.0 \mathrm{w} / \mathrm{ft}^{2}\end{array}$ & $\begin{array}{l}0.8 \mathrm{w} / \mathrm{ft}^{2} \\
1.0 \mathrm{w} / \mathrm{ft}^{2}\end{array}$ & $\begin{array}{l}0.8 \mathrm{w} / \mathrm{ft}^{2} \\
1.0 \mathrm{w} / \mathrm{ft}^{2}\end{array}$ \\
\hline \multicolumn{6}{|c|}{ 505.6 Exterior Lighting } \\
\hline & Multifamily building & $\begin{array}{l}\text { Parking: } 0.06 \\
\text { W/ft } \\
\text { Façade: } 0.1 \mathrm{~W} / \mathrm{ft}^{2}\end{array}$ & $\begin{array}{l}\text { Parking: } 0.15 \mathrm{~W} / \mathrm{ft}^{2} \\
\text { Façade: } 0.2 \mathrm{~W} / \mathrm{ft}^{2}\end{array}$ & & $\begin{array}{l}\text { Parking: } 0.15 \mathrm{~W} / \mathrm{ft}^{2} \\
\text { Façade: } 0.2 \mathrm{~W} / \mathrm{ft}^{2}\end{array}$ \\
\hline & $\begin{array}{l}\text { Retail } \\
\text { Warehouse } \\
\text { Small Office }\end{array}$ & $\begin{array}{l}\text { Parking: } 0.10 \\
\text { W/ft }{ }^{2} \\
\text { Main entries: } 30 \\
\text { W/ft } \\
\text { Other doors: } 20 \\
\text { W/ft } \\
\text { Façade: } 0.15 \\
\text { W/ft } \\
\end{array}$ & $\begin{array}{l}\text { Parking: } 0.15 \mathrm{~W} / \mathrm{ft}^{2} \\
\text { Main entries: } 30 \mathrm{~W} / \mathrm{ft} \\
\text { Other doors: } 20 \mathrm{~W} / \mathrm{ft} \\
\text { Façade: } 0.2 \mathrm{~W} / \mathrm{ft}^{2}\end{array}$ & $\begin{array}{l}\text { No specific LPD } \\
\text { requirements. }\end{array}$ & $\begin{array}{l}\text { Parking: } 0.15 \mathrm{~W} / \mathrm{ft}^{2} \\
\text { Main entries: } 30 \mathrm{~W} / \mathrm{ft} \\
\text { Other doors: } 20 \mathrm{~W} / \mathrm{ft} \\
\text { Façade: } 0.2 \mathrm{~W} / \mathrm{ft}^{2}\end{array}$ \\
\hline
\end{tabular}




\section{Energy Analysis}

The impact of code requirements are quantified by analyzing building models representing the envelope, lighting and mechanical requirements identified in the comparison Tables 1 and 2. DOE Commercial Reference Building models for four representative building types were used for the analysis. A brief description of each e building type is given below:

1. Residential - Multifamily/Mid-rise apartment building (33,700 sf): This is a four story building with $15 \%$ window-wall ratio. This building is assumed to have steel frame walls, metal deck roof with insulation above deck and a slab-on-grade floor. Each apartment unit is assumed to have a packaged airconditioning unit with a gas furnace and a 20-gallon electric service water heating system. More details of the thermal model with the baseline properties are listed in Appendix-A, Table A-1.

2. Nonresidential - Medium office building (53,600 sf): This is a three-story building with a $33 \%$ window-wall ratio. This building is assumed to have steel frame walls, metal deck roof with insulation above deck and a slab-on-grade floor. The HVAC system is multi-zone variable air volume (VAV) system with electric reheat, and packaged air-conditioner with gas furnace. Service water heating is assumed to be 260-gallon gas water heater. Further details on the energy model are provided in Appendix-A, Table A-2.

3. Nonresidential - Small retail / strip mall building (22,500 sf): This is a single-story strip mall building with $10 \%$ window-wall ratio. The floor plan is assumed to have ten store spaces including two large stores of $3750 \mathrm{sf}$ each and eight smaller stores of $1875 \mathrm{sf}$ each. This building is assumed to have steel frame walls, metal deck rook with insulation above deck and a slab-on-grade floor. Each store is assumed to have a rooftop packaged air-conditioning unit with a gas furnace and a 6 gallon natural gas service water heating system.

4. Semiheated - Large warehouse building (52,045 sf): This is a non-refrigerated warehouse building with an office area, fine storage and bulk storage areas. This building is assumed to have metal building walls, metal deck rook with insulation above deck and a slab-on-grade floor. The office area is conditioned by a packaged air conditioning unit with gas furnace and the storage areas are heated with a natural gas unit heater. Service water heating is assumed to be a 20 gallon electric storage water heater.

Hourly energy simulation of the building model was performed for each of the four codes: 2003 IECC, 2006 IECC, 2009 IECC and ASHRAE 90.1-2007. EnergyPlus simulation software was used for the analysis. A typical design day simulation was done to determine the HVAC equipment size, and then the annual simulation was run to determine the building energy use. The total building site energy is extracted from the simulation results for code comparisons.

To assess the economic impacts of the code requirements, current utility tariffs for Mesa City ${ }^{\mathrm{b}}$ are used to calculate the total annual energy cost. Table 3 shows the fuel prices, demand charges and rate structure used, assuming a basic rate plan.

\footnotetext{
${ }^{\mathrm{b}}$ http://www.mesaaz.gov/custserv/pdf/Utilityratebook.pdf
} 
Table 3: Utility tariffs used for Mesa City (2010-2011)

\begin{tabular}{|c|c|c|c|c|}
\hline & \multicolumn{2}{|c|}{ Nonresidential } & \multicolumn{2}{|c|}{ Residential } \\
\hline & Gas & Electricity & Gas & Electricity \\
\hline \multirow[t]{2}{*}{ Monthly Charge } & Winter: $\$ 37.77 /$ month & \multirow[t]{2}{*}{$\$ 6.22 /$ month } & Winter: $\$ 12.3 /$ month & \multirow[t]{2}{*}{$\$ 5.71 /$ month } \\
\hline & Summer:\$28.32/month & & Summer:\$9.44/month & \\
\hline \multirow[t]{8}{*}{ Energy Charge } & Winter: & Winter: & Winter: & Winter: \\
\hline & $\begin{array}{l}<1200 \text { therm: } \\
\$ 0.5579 / \text { therm }\end{array}$ & $\begin{array}{l}\text { <15000kWh: } \\
\$ 0.07595 / \mathrm{kWh}\end{array}$ & $\begin{array}{l}<25 \text { therm: } \\
\$ 0.6522 / \text { therm }\end{array}$ & $\begin{array}{l}\text { <800kWh: } \\
\$ 0.07065 / \mathrm{kWh}\end{array}$ \\
\hline & \multirow[t]{2}{*}{$\begin{array}{l}\text { Remaining: } \\
\$ 0.4462 / \text { therm }\end{array}$} & $\begin{array}{l}\text { >15000kWh\& } \\
<60000 \text { kWh: } \\
\$ 0.05912 / \text { kWh }\end{array}$ & $\begin{array}{l}\text { Remaining: } \\
\$ 0.4806 / \text { therm }\end{array}$ & $\begin{array}{l}\text { Remaining: } \\
\$ 0.04933 \text { kWh }\end{array}$ \\
\hline & & $\begin{array}{l}\text { Remaining: } \\
\text { \$0.0428 kWh }\end{array}$ & & \\
\hline & Summer: & Summer: & Summer: & Summer: \\
\hline & $\begin{array}{l}<1200 \text { therm: } \\
\$ 0.5151 / \text { therm }\end{array}$ & $\begin{array}{l}<15000 \text { kWh: } \\
\$ 0.08941 / \text { kWh }\end{array}$ & $\begin{array}{l}<25 \text { therm: } \\
\$ 0.6522 / \text { therm }\end{array}$ & $\begin{array}{l}<1200 \text { kWh: } \\
\$ 0.08768 / \text { kWh }\end{array}$ \\
\hline & \multirow[t]{2}{*}{$\begin{array}{l}\text { Remaining: } \\
\$ 0.3089 / \text { therm }\end{array}$} & $\begin{array}{l}\text { >15000 kWh \& } \\
<60000 \text { kWh: }\end{array}$ & \multirow[t]{2}{*}{$\begin{array}{l}\text { Remaining: } \\
\$ 0.2114 / \text { therm }\end{array}$} & \multirow[t]{2}{*}{$\begin{array}{l}\text { Remaining: } \\
\$ 0.08462 \text { kWh }\end{array}$} \\
\hline & & $\begin{array}{l}\text { Remaining: } \\
\$ 0.05351 \text { kWh }\end{array}$ & & \\
\hline \multirow[t]{2}{*}{ Demand Charge } & \multirow[t]{2}{*}{ N.A. } & Winter: >50W: & \multirow[t]{2}{*}{ N.A. } & \multirow[t]{2}{*}{ N.A. } \\
\hline & & $\begin{array}{l}\text { Summer: }>50 W: \\
\$ 3.9168 / W\end{array}$ & & \\
\hline
\end{tabular}

In addition to calculating the annual energy consumption and cost savings, the analysis also includes estimates for carbon emissions based on state-average $\mathrm{CO}_{2}$ emission factors available in the EnergyPlus data set. For the state of Arizona, $\mathrm{CO}_{2}$ emission factors assumed are: $50.23 \mathrm{~g} / \mathrm{MJ}$ for natural gas and $132.3 \mathrm{~g} / \mathrm{MJ}$ for electricity.

The energy simulation results are summarized in Table 4 for all the building types and codes considered in this study. This summary includes total annual electricity consumption, natural gas consumption, electricity demand, annual energy cost and $\mathrm{CO}_{2}$ emissions. It can be observed that the overall savings are significantly higher for residential construction than nonresidential or semiheated buildings. This can be attributed to the increased insulation requirements for above-grade envelope components for residential buildings in the 2009 IECC and ASHRAE 90.1. 
Table 4: Detailed Simulation Results

\begin{tabular}{|c|c|c|c|c|c|c|c|}
\hline \multicolumn{8}{|c|}{ Mesa, AZ -- Energy End Use Detailed Results } \\
\hline \multirow[b]{2}{*}{$\begin{array}{c}\text { Building } \\
\text { Prototype }\end{array}$} & \multirow[b]{2}{*}{ Code } & \multicolumn{6}{|c|}{ Energy Use Intensity } \\
\hline & & $\begin{array}{c}\text { Electricity } \\
\text { (kWh/sf/yr) }\end{array}$ & $\begin{array}{l}\text { Natural Gas } \\
\text { (kBtu/sf/yr) }\end{array}$ & $\begin{array}{c}\text { EUI } \\
\text { (kBtu/sf) }\end{array}$ & $\begin{array}{l}\text { Elec.Demand } \\
\qquad(\mathrm{kW})\end{array}$ & $\begin{array}{l}\text { Utility } \\
\text { Cost } \\
(\$)\end{array}$ & $\begin{array}{c}\mathrm{CO}_{2} \\
\text { Emission } \\
\text { (tons) }\end{array}$ \\
\hline \multirow{4}{*}{ Multifamily } & 2003 IECC & 14.1 & 1.6 & 49.7 & 108.3 & $\$ 33,734$ & 230.7 \\
\hline & 2006 IECC & 12.0 & 1.0 & 42.0 & 87.6 & $\$ 28,670$ & 196.4 \\
\hline & 2009 IECC & 11.0 & 0.7 & 38.4 & 75.3 & $\$ 26,091$ & 179.8 \\
\hline & ASHRAE 90.1-2007 & 11.0 & 0.6 & 38.1 & 74.8 & $\$ 25,890$ & 178.5 \\
\hline \multirow{4}{*}{ Medium office } & 2003 IECC & 14.5 & 2.4 & 52.0 & 304.2 & $\$ 61,813$ & 382.2 \\
\hline & 2006 IECC & 13.7 & 2.5 & 49.1 & 285.1 & $\$ 58,363$ & 360.0 \\
\hline & 2009 IECC & 13.1 & 2.5 & 47.0 & 276.8 & $\$ 56,065$ & 344.3 \\
\hline & ASHRAE 90.1-2007 & 13.4 & 2.5 & 48.3 & 276.8 & $\$ 57,303$ & 353.8 \\
\hline \multirow{4}{*}{ Small retail } & 2003 IECC & 20.2 & 4.1 & 72.9 & 164.1 & $\$ 36,674$ & 222.9 \\
\hline & 2006 IECC & 18.9 & 3.3 & 68.0 & 152.3 & $\$ 34,394$ & 208.7 \\
\hline & 2009 IECC & 17.8 & 3.0 & 63.7 & 144.3 & $\$ 32,450$ & 195.7 \\
\hline & ASHRAE 90.1-2007 & 18.2 & 3.0 & 65.2 & 144.3 & $\$ 33,071$ & 200.5 \\
\hline \multirow{4}{*}{ Large warehouse } & 2003 IECC & 6.9 & 2.1 & 25.8 & 129.0 & $\$ 29,608$ & 179.5 \\
\hline & 2006 IECC & 5.9 & 1.5 & 21.5 & 96.5 & $\$ 24,788$ & 150.4 \\
\hline & 2009 IECC & 5.6 & 1.4 & 20.5 & 92.1 & $\$ 23,717$ & 143.3 \\
\hline & ASHRAE 90.1-2007 & 5.8 & 1.5 & 21.2 & 95.1 & $\$ 24,482$ & 148.5 \\
\hline
\end{tabular}

Table 5: Energy Cost Summary (\$/sf/year)

\begin{tabular}{|l|c|c|c|c|}
\hline \multicolumn{5}{|c|}{ Mesa, AZ - Annual Energy Cost Comparison (\$/sf) } \\
\hline Building Prototype & $\mathbf{2 0 0 3}$ IECC & $\mathbf{2 0 0 6}$ IECC & 2009 IECC & ASHRAE 90.1-2007 \\
\hline Multifamily & 1.00 & 0.85 & 0.77 & 0.77 \\
\hline Medium office & 1.15 & 1.09 & 1.05 & 1.07 \\
\hline Small retail & 1.63 & 1.53 & 1.44 & 1.47 \\
\hline Large warehouse & 0.57 & 0.48 & 0.46 & 0.47 \\
\hline
\end{tabular}




\section{Conclusions}

Assuming that the current buildings in Mesa, AZ are complying with the 2003 IECC or the 2006 IECC commercial building requirements, Tables 6 and 7 show the comparison of energy savings and $\mathrm{CO}_{2}$ emission reductions if these are built to comply with the 2009 IECC and ASHRAE Standard 90.1-2007, given the assumptions used in the analysis.

Table 6: Energy Use and Cost Savings Comparison (percentage)

\begin{tabular}{|c|c|c|c|c|c|c|c|c|}
\hline \multirow[b]{4}{*}{ Building Prototype } & \multicolumn{8}{|c|}{ Mesa, AZ -- Energy End Use Percentage Savings } \\
\hline & \multicolumn{4}{|c|}{2009 IECC } & \multicolumn{4}{|c|}{ ASHRAE 90.1-2007 } \\
\hline & \multicolumn{2}{|c|}{ vs. 2003 IECC } & \multicolumn{2}{|c|}{ VS. 2006 IECC } & \multicolumn{2}{|c|}{ VS. 2003 IECC } & \multicolumn{2}{|c|}{ vs. 2006 IECC } \\
\hline & EUI & Cost & EUI & Cost & EUI & Cost & EUI & Cost \\
\hline Multifamily & $29.4 \%$ & $29.3 \%$ & $9.5 \%$ & $9.9 \%$ & $30.6 \%$ & $30.3 \%$ & $10.5 \%$ & $10.7 \%$ \\
\hline Medium office & $10.6 \%$ & $10.3 \%$ & $4.4 \%$ & $4.1 \%$ & $7.7 \%$ & $7.9 \%$ & $1.7 \%$ & $1.9 \%$ \\
\hline Small retail & $14.5 \%$ & $13.0 \%$ & $6.8 \%$ & $6.0 \%$ & $11.8 \%$ & $10.9 \%$ & $4.3 \%$ & $4.0 \%$ \\
\hline Large warehouse & $26.1 \%$ & $24.8 \%$ & $5.0 \%$ & $4.5 \%$ & $21.6 \%$ & $20.9 \%$ & $1.2 \%$ & $1.3 \%$ \\
\hline
\end{tabular}

Table 7: $\mathrm{CO}_{2}$ Emission Reduction Comparison (percentage)

\begin{tabular}{|c|c|c|c|c|}
\hline \multicolumn{5}{|c|}{ Mesa, $\mathrm{AZ}-\mathrm{CO}_{2}$ Emission Reduction } \\
\hline \multirow[b]{2}{*}{ Building Prototype } & \multicolumn{2}{|c|}{2009 IECC } & \multicolumn{2}{|c|}{ ASHRAE 90.1-2007 } \\
\hline & vs. 2003 IECC & vs. IECC 2006 & vs. 2003 IECC & vs. IECC 2006 \\
\hline Multifamily & $22.00 \%$ & $8.45 \%$ & $22.67 \%$ & $9.15 \%$ \\
\hline Medium office & $9.92 \%$ & $4.35 \%$ & $7.44 \%$ & $1.70 \%$ \\
\hline Small retail & $12.19 \%$ & $6.23 \%$ & $10.05 \%$ & $3.94 \%$ \\
\hline Large warehouse & $20.18 \%$ & $4.72 \%$ & $17.31 \%$ & $1.28 \%$ \\
\hline
\end{tabular}

Based on the analysis, it can be observed that:

- Multifamily buildings built to the 2009 IECC or ASHRAE Standard 90.1-2007 would use 30\% less energy and save $30 \%$ in energy cost compared to the 2003 IECC, and reduce $\mathrm{CO}_{2}$ emissions by $20 \%$. They would use $10 \%$ less energy and save $10 \%$ in energy cost compared to the 2006 IECC, and reduce $\mathrm{CO}_{2}$ emissions between 8 and $9 \%$.

- All nonresidential buildings built to the 2009 IECC save between 10 and 25\% energy and cost compared to the 2003 IECC and reduce $\mathrm{CO}_{2}$ emissions by 10-20\% depending on the building type. They would save between 4 and 6\% energy and cost, and reduce $\mathrm{CO}_{2}$ emissions by 5\% compared to the 2006 IECC.

- All nonresidential buildings built to ASHRAE Standard 90.1-2007 have similar but relatively lower savings than buildings complying with 2009 IECC. 


\section{Appendix A - Prototype Building Descriptions}

Table A-1: Residential Prototype Building Characteristics

\begin{tabular}{|c|c|}
\hline \multicolumn{2}{|c|}{ Multifamily Building Characteristics } \\
\hline \multicolumn{2}{|l|}{ General } \\
\hline Building type & Mid-rise Apartment \\
\hline Total floor area & $33,700 \mathrm{sqft}$ \\
\hline Building shape & Rectangle $(152 \mathrm{ft} \times 55.5 \mathrm{ft})$ \\
\hline Aspect ratio & 2.74 \\
\hline Number of floors & 4 \\
\hline Window fraction & $15 \%$ \\
\hline Window shading & None \\
\hline Thermal zoning & $\begin{array}{l}\text { Ground floor: } 7 \text { apartments and } 1 \text { lobby with equivalent apartment area } \\
\text { Floors above: } 8 \text { apartments with corridor in center } \\
\text { Zone depth is } 25 \mathrm{ft} \text { for each apartment from side walls and each apt is } 25^{\prime} \\
\times 38^{\prime}\left(950 \mathrm{ft}^{2}\right) \text {. }\end{array}$ \\
\hline Floor to floor height (feet) & $10 \mathrm{ft}$ \\
\hline Floor to ceiling height (feet) & $10 \mathrm{ft}$ (no drop down ceiling modeled) \\
\hline Glazing sill height (feet) & $3 \mathrm{ft}$. (14 ft. wide $\times 4 \mathrm{ft}$. high) \\
\hline Exterior walls & Steel-framed wall \\
\hline Roof & Insulation entirely above deck, metal deck roof \\
\hline Foundation & 8 inch concrete slab-on-grade floors (unheated) \\
\hline Interior partitions & $2 \times 4$ uninsulated stud wall \\
\hline Internal mass & 6 inches standard wood $\left(16.6 \mathrm{lb} / \mathrm{ft}^{2}\right)$ \\
\hline Infiltration & $\begin{array}{l}\text { Peak: } 0.2016 \mathrm{cfm} / \mathrm{sf} \text { of above grade exterior wall surface area (when fans } \\
\text { turn off) } \\
\text { Off Peak: } 25 \% \text { of peak infiltration rate (when fans turn on) }\end{array}$ \\
\hline \multicolumn{2}{|l|}{ Internal Loads \& Schedules } \\
\hline Lighting power density $\left(\mathrm{W} / \mathrm{ft}^{2}\right)$ & $\begin{array}{l}\text { Apartment units: } 0.36 \mathrm{~W} / \mathrm{ft}^{2} \\
\text { Corridor: } 0.5 \mathrm{~W} / \mathrm{ft}^{2} \\
\text { Office: } 1.1 \mathrm{~W} / \mathrm{ft}^{2}\end{array}$ \\
\hline Plug load power density $\left(\mathrm{W} / \mathrm{ft}^{2}\right)$ & Building average, $0.62 \mathrm{~W} / \mathrm{ft}^{2}$ \\
\hline Occupancy & 78 Total (2.5 person per apartment unit) \\
\hline
\end{tabular}




\section{Table A-1: Residential Prototype Building Characteristics (continued)}

\begin{tabular}{|l|l|}
\hline \multicolumn{2}{|l|}{ HVAC } \\
\hline \multicolumn{2}{|l|}{ System Type } \\
\hline Heating type & Gas furnace inside the packaged air conditioning unit \\
\hline Cooling type & Split DX System \\
\hline Distribution and terminal units & Constant volume \\
\hline HVAC Control & $75^{\circ} \mathrm{F}$ Cooling/70 ${ }^{\circ} \mathrm{F}$ Heating \\
\hline Thermostat setpoint & No setback \\
\hline Thermostat setback & Maximum $110^{\circ} \mathrm{F}$, Minimum $52^{\circ} \mathrm{F}$ \\
\hline Supply air temperature & 20 cfm/person \\
\hline Ventilation & No \\
\hline Demand control ventilation & No \\
\hline Energy recovery & \multicolumn{2}{|l}{} \\
\hline Supply Fan & Constant air volume \\
\hline Fan type & $70 \%$ \\
\hline Supply fan total efficiency (\%) & 0.2 inch water \\
\hline Supply fan pressure drop & Storage tank \\
\hline Service Water Heating & Electricity \\
\hline SWH type & $100 \%$ \\
\hline Fuel type & 20 (one per unit) \\
\hline Thermal efficiency (\%) & $120^{\circ} \mathrm{F}$ \\
\hline Tank volume (gal) & Not modeled \\
\hline Water temperature setpoint & \multicolumn{2}{|l}{} \\
\hline MisC. & Exterior Lighting \\
\hline
\end{tabular}


Table A-2: Nonresidential Prototype Building Characteristics

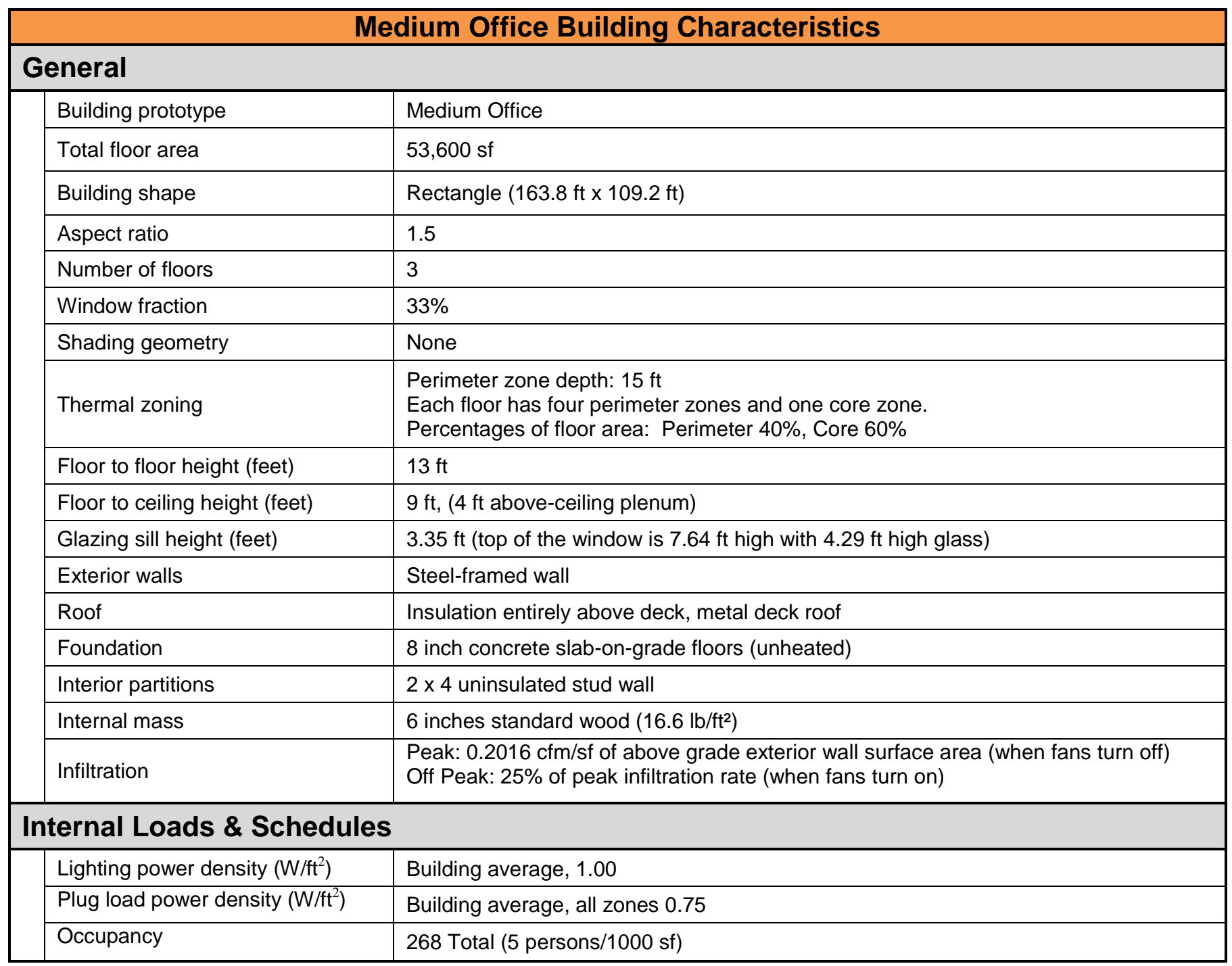


Table A-2: Nonresidential Prototype Building Characteristics (continued)

\begin{tabular}{|c|c|}
\hline \multicolumn{2}{|l|}{ HVAC } \\
\hline \multicolumn{2}{|l|}{ System Type } \\
\hline Heating type & Gas furnace inside the packaged air conditioning unit \\
\hline Cooling type & Packaged air conditioning unit \\
\hline Distribution and terminal units & $\begin{array}{l}\text { VAV terminal box with damper and electric reheating coil } \\
\text { Zone control type: minimum supply air at } 30 \% \text { of the zone design peak supply air. }\end{array}$ \\
\hline \multicolumn{2}{|l|}{ HVAC Control } \\
\hline Thermostat setpoint & $75^{\circ} \mathrm{F}$ Cooling $/ 70^{\circ} \mathrm{F}$ Heating \\
\hline Thermostat setback & $80^{\circ} \mathrm{F}$ Cooling $/ 60^{\circ} \mathrm{F}$ Heating \\
\hline Supply air temperature & Maximum $110^{\circ} \mathrm{F}$, Minimum $52^{\circ} \mathrm{F}$ \\
\hline Ventilation & $20 \mathrm{cfm} /$ person \\
\hline Demand control ventilation & No \\
\hline Energy recovery & No \\
\hline \multicolumn{2}{|l|}{ Supply Fan } \\
\hline Fan type & Variable air volume \\
\hline Supply fan total efficiency (\%) & $57 \%$ to $60 \%$ depending on the fan motor size \\
\hline Supply fan pressure drop & 4"-6.3" water \\
\hline \multicolumn{2}{|l|}{ Service Water Heating } \\
\hline SWH type & Storage tank \\
\hline Fuel type & Natural gas \\
\hline Thermal efficiency (\%) & $80 \%$ \\
\hline Tank volume (gal) & 260 \\
\hline Water temperature setpoint & $120^{\circ} \mathrm{F}$ \\
\hline \multicolumn{2}{|l|}{ Misc. } \\
\hline \multicolumn{2}{|l|}{ Exterior Lighting } \\
\hline Peak power & $2730 \mathrm{~W}$ \\
\hline
\end{tabular}


Table A-3: Small Retail Prototype Building Characteristics

\begin{tabular}{|c|c|}
\hline \multicolumn{2}{|r|}{ Small Retail Building Characteristics } \\
\hline \multicolumn{2}{|l|}{ General } \\
\hline Building prototype & Small retail \\
\hline Total floor area & $\begin{array}{l}22,500 \mathrm{ft}^{2} \\
(10 \text { stores, including } 2 \text { large stores of } 3750 \text { sqft, and } 8 \text { small stores of } 1875 \\
\text { sqft) }\end{array}$ \\
\hline Building shape & Rectangle \\
\hline Aspect ratio & 4 (0.33 for small stores and 0.67 for large stores) \\
\hline Number of floors & 1 \\
\hline Window fraction & $10.5 \%$ \\
\hline Shading geometry & none \\
\hline Thermal zoning & One zone per store \\
\hline Floor to floor height (feet) & $17 \mathrm{ft}$ \\
\hline Floor to ceiling height (feet) & $17 \mathrm{ft}$ \\
\hline Glazing sill height (feet) & $3.00 \mathrm{ft}$ (top of the window is $8 \mathrm{ft}$ high) \\
\hline Exterior walls & Steel-framed wall \\
\hline Roof & Insulation entirely above deck, metal deck roof \\
\hline Foundation & 6 inch concrete slab-on-grade floors (unheated) \\
\hline Interior partitions & 2x4 steel-frame with gypsum board \\
\hline Internal mass & $\begin{array}{l}6 \text { inch wood } \\
\text { total mass area: } 45,000\left(\mathrm{ft}^{2}\right) \\
\text { includes } 2 \text { large stores }\left(7500 \mathrm{ft}^{2} \text { each }\right) \text { and } 8 \text { small stores }\left(3750 \mathrm{ft}^{2} \text { each }\right)\end{array}$ \\
\hline Infiltration & $\begin{array}{l}\text { Peak: } 0.2016 \mathrm{cfm} / \mathrm{sf} \text { of above grade exterior wall surface area (when fans turn } \\
\text { off) } \\
\text { Off Peak: } 25 \% \text { of peak infiltration rate (when fans turn on) }\end{array}$ \\
\hline \multicolumn{2}{|l|}{ Internal Loads \& Schedules } \\
\hline Lighting power density $\left(\mathrm{W} / \mathrm{ft}^{2}\right)$ & $1.5 \mathrm{~W} / \mathrm{ft}^{2}$ \\
\hline Plug load power density $\left(\mathrm{W} / \mathrm{ft}^{2}\right)$ & Building average, all zones $0.4 \mathrm{~W} / \mathrm{ft}^{2}$ \\
\hline Occupancy & 30 in each large store, and 15 in each small store \\
\hline
\end{tabular}


Table A-3: Small Retail Prototype Building Characteristics (continued)

\begin{tabular}{|c|c|}
\hline \multicolumn{2}{|l|}{ HVAC } \\
\hline \multicolumn{2}{|l|}{ System Type } \\
\hline Heating type & Gas furnace inside the packaged air conditioning unit \\
\hline Cooling type & Packaged air conditioning unit \\
\hline Distribution and terminal units & $\begin{array}{l}10 \text { single-zone rooftop units with constant air volume system. } \\
\text { One unit serving one store. }\end{array}$ \\
\hline \multicolumn{2}{|l|}{ HVAC Control } \\
\hline Thermostat setpoint & $75^{\circ} \mathrm{F}$ Cooling $/ 70^{\circ} \mathrm{F}$ Heating \\
\hline Thermostat setback & $85^{\circ} \mathrm{F}$ Cooling $/ 60^{\circ} \mathrm{F}$ Heating \\
\hline Supply air temperature & Maximum $104^{\circ} \mathrm{F}$, Minimum $55^{\circ} \mathrm{F}$ \\
\hline Ventilation & $20 \mathrm{cfm} /$ person \\
\hline Demand control ventilation & No \\
\hline Energy recovery & No \\
\hline \multicolumn{2}{|l|}{ Supply Fan } \\
\hline Fan Type & Constant air volume \\
\hline Supply fan total efficiency (\%) & $60 \%$ to $62 \%$ depending on the fan motor size \\
\hline Supply fan pressure drop & Various depending on the fan supply air cfm \\
\hline \multicolumn{2}{|l|}{ Service Water Heating } \\
\hline SWH type & Storage tank \\
\hline Fuel type & Natural gas \\
\hline Thermal efficiency (\%) & $78.40 \%$ \\
\hline Tank volume (gal) & 6 \\
\hline Water temperature setpoint & $120^{\circ} \mathrm{F}$ \\
\hline \multicolumn{2}{|l|}{ Misc. } \\
\hline \multicolumn{2}{|l|}{ Exterior Lighting } \\
\hline Peak power & See Table.2 \\
\hline
\end{tabular}


Table A-4: Semiheated Prototype Building Characteristics

\begin{tabular}{|c|c|}
\hline \multicolumn{2}{|c|}{ Large Warehouse Building Characteristics } \\
\hline \multicolumn{2}{|l|}{ General } \\
\hline Building type & Non-refrigerated warehouse \\
\hline Total floor area & $52,045 \mathrm{sqft}$ \\
\hline Building shape & Rectangle \\
\hline Aspect ratio & 2.2 \\
\hline Number of floors & 1 \\
\hline Window fraction & $\begin{array}{l}\text { Storage Area: No Windows; } \\
\text { Office Area: } 12 \% \text { View windows; } \\
\text { Overall: } 0.71 \% \text {; } \\
\text { North: } 0.76 \% ; \\
\text { East: } 0.0 \% \text {; } \\
\text { South: } 0.0 \% \text {; } \\
\text { West: } 2.86 \%\end{array}$ \\
\hline Shading geometry & None \\
\hline Thermal zoning & $\begin{array}{l}\text { Office: } 2549.76 \mathrm{ft}^{2} \\
\text { Fine Storage: } 14,998.54 \mathrm{ft}^{2} \\
\text { Bulk Storage: } 34496.61 \mathrm{ft}^{2} \text { (semi-heated) }\end{array}$ \\
\hline Floor to floor height & $28 \mathrm{ft}$ \\
\hline Floor to ceiling height (feet) & 14ft (for the office area only) \\
\hline Glazing sill height & $3 \mathrm{ft}$ (top of the window is $8 \mathrm{ft}$ high) \\
\hline Exterior walls & MetalBuilding \\
\hline Roof & MetalBuilding \\
\hline Foundation & 6" concrete slab-on-Grade \\
\hline Interior partitions & double layer of sypsum board with an exterior layer of stucco \\
\hline Internal mass & $\begin{array}{l}\text { defined as material with properties: } \\
\text { density: } 12.5 \mathrm{lb} / \mathrm{sf} \\
\text { thickness: } 8 \mathrm{ft} \\
\text { Internal mass surface area: } 64889.66 \mathrm{ft}^{2}\end{array}$ \\
\hline Infiltration & $\begin{array}{l}\text { Office: } 0.078 \mathrm{cfm} / \mathrm{ft}^{2} \\
\text { Fine storage: } 0.080 \mathrm{cfm} / \mathrm{ft}^{2} \\
\text { Bulk storage: } 4793.56 \mathrm{cfm}\end{array}$ \\
\hline \multicolumn{2}{|l|}{ Internal Loads \& Schedules } \\
\hline \multicolumn{2}{|l|}{ Lighting } \\
\hline Average power density $\left(\mathrm{W} / \mathrm{ft}^{2}\right)$ & $\begin{array}{l}\text { Fine and bulk area: } 0.80 \mathrm{w} / / \mathrm{ft}^{2} \\
\text { Office area: } 1.00 \mathrm{w} / \mathrm{ft}^{2}\end{array}$ \\
\hline Daylighting controls & Daylight control: bulk storage area \\
\hline Occupancy sensors & No \\
\hline Plug Load power density (W/ft $\left.{ }^{2}\right)$ & $\begin{array}{l}\text { Office: } 0.75 \mathrm{w} / / \mathrm{ft}^{2} \\
\text { Bulk storage: } 0.24 \mathrm{w} / / \mathrm{ft}^{2}\end{array}$ \\
\hline Occupancy & 5 (in the office) \\
\hline
\end{tabular}


Table A-4: Semiheated Prototype Building Characteristics (continued)

\begin{tabular}{|c|c|}
\hline \multicolumn{2}{|l|}{ HVAC } \\
\hline \multicolumn{2}{|l|}{ System Type } \\
\hline Heating type & Gas-fired furnace \\
\hline Cooling type & DX cooling coil \\
\hline Distribution and terminal units & Direct uncontrolled air \\
\hline \multicolumn{2}{|l|}{ HVAC Control } \\
\hline Thermostat setpoint & $\begin{array}{l}\text { Fine storage: } 80^{\circ} \mathrm{F} \text { Cooling/ } 60^{\circ} \mathrm{F} \text { Heating; } \\
\text { Office Area: } 75^{\circ} \mathrm{F} \text { Cooling/ } 70^{\circ} \mathrm{F} \text { Heating; } \\
\text { Bulk Storage: } 50^{\circ} \mathrm{F} \text { Heating; }\end{array}$ \\
\hline Thermostat setback & Office Area: $85^{\circ} \mathrm{F}$ Cooling $/ 60^{\circ} \mathrm{F}$ Heating \\
\hline Supply air temperature & Maximum $110^{\circ} \mathrm{F}$, Minimum $55^{\circ} \mathrm{F}$ \\
\hline Ventilation & $\begin{array}{l}\text { bulk storage: } 80009 \mathrm{cfm} \text { (exhaust); } 2000 \mathrm{cfm} \text { (natural) } \\
\text { Office: } 0.085 \mathrm{cfm} / \mathrm{ft}^{2} \\
\text { Storage: } 0.06 \mathrm{cfm} / \mathrm{ft}^{2}\end{array}$ \\
\hline Demand control ventilation & No \\
\hline Energy recovery & No \\
\hline \multicolumn{2}{|l|}{ Supply Fan } \\
\hline Fan type & Constant air volume \\
\hline Supply fan total efficiency (\%) & $\begin{array}{l}\text { Office Area: fan efficiency } 54.6 \% \text {, motor efficiency } 84 \% \text {; } \\
\text { fine storage: fan efficiency } 56.875 \% \text {, motor efficiency } 87.5 \text {; } \\
\text { bulk storage unit heater: fan efficiency } 53.6 \% \text {, motor efficiency } 82.5 \%\end{array}$ \\
\hline Supply fan pressure drop & $\begin{array}{l}\text { Office Area: } 2.5 \text { " Water } \\
\text { Fine storage: } 2.5 \text { " water } \\
\text { Bulk storage: } 0.2 \text { " water }\end{array}$ \\
\hline \multicolumn{2}{|l|}{ Service Water Heating } \\
\hline SWH type & Electric storage water heater \\
\hline Fuel type & Electricity \\
\hline Thermal efficiency (\%) & $100 \%$ \\
\hline Tank volume (gal) & 20 \\
\hline Water temperature setpoint & $120^{\circ} \mathrm{F}$ \\
\hline \multicolumn{2}{|l|}{ Misc. } \\
\hline \begin{tabular}{l|l} 
Exterior lighting peak power
\end{tabular} & 4800 watts \\
\hline
\end{tabular}




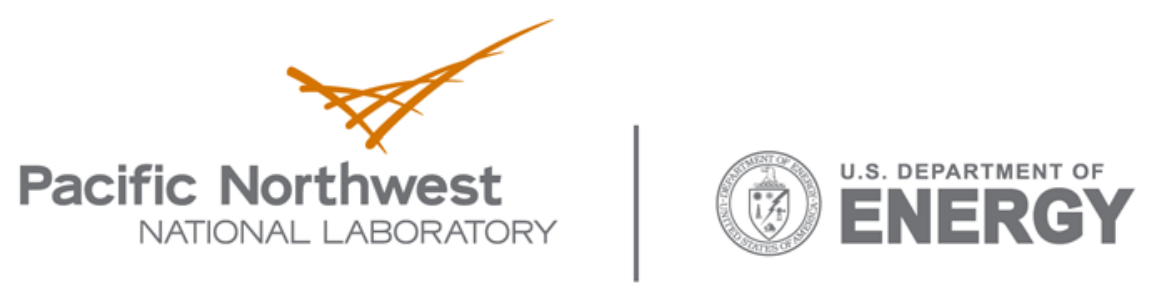

902 Battelle Boulevard

P.O. Box 999

Richland, WA 99352

1-888-375-PNNL (7665)

www.pnl.gov 